\title{
Florescimento no Trabalho: Impacto do Perdão Disposicional e do Suporte Social Emocional
}

\author{
Solange Maia da Silva Chaves ${ }^{1}$ \\ ${ }^{1}$ Universidade Salgado de Oliveira, RJ, Brasil. \\ Michelle Morelo Pereira ${ }^{2}$ \\ ${ }^{2}$ Faculdades Integradas Maria Thereza, RJ, Brasil.
}

\author{
Maria Cristina Ferreira ${ }^{1}$ \\ ${ }^{1}$ Universidade Salgado de Oliveira, RJ, Brasil. \\ Clarissa Pinto Pizarro de Freitas ${ }^{3}$ \\ ${ }^{3}$ Universidade Salgado de Oliveira, RJ, Brasil.
}

Resumo: O florescimento no trabalho tem despertado interesse crescente dos estudiosos, no contexto do modelo de Demandas e Recursos do Trabalho. O objetivo deste estudo foi investigar as relações de um recurso pessoal (perdão disposicional) e de um recurso do trabalho (suporte social emocional) com o florescimento no trabalho de enfermeiros, bem como avaliar o papel mediador do suporte social emocional nessas relações. A amostra foi composta por 515 trabalhadores de enfermagem. Os resultados da modelagem de equações estruturais apontaram que o perdão disposicional e o suporte social emocional relacionaram-se positivamente ao florescimento no trabalho. Ademais, o suporte social emocional mediou parcialmente a relação entre o perdão disposicional e o florescimento no trabalho. A escolaridade também exerceu impacto sobre o florescimento no trabalho. O modelo de mediação parcial apresentou índices de ajuste adequados. Os resultados demonstraram a relevância do suporte social emocional para o florescimento no trabalho, assim como sua relação com o perdão disposicional. O estudo avançou, portanto, na explicação de alguns dos fatores responsáveis pelo florescimento no trabalho de enfermeiros, categoria profissional de grande importância para a sociedade em geral.

Palavras-chave: Florescimento no Trabalho, Suporte Social Emocional, Perdão Disposicional, Bem-Estar no Trabalho.

\section{Flourishing at Work: Impact of Dispositional Forgiveness and Emotional Social Support}

\begin{abstract}
The flourishing at work has attracted growing interest from scholars, in the context of the Job Demands and Resources model. The aim of this study was to investigate the relationships of a personal resource (dispositional forgiveness) and a job resource (emotional social support) with the flourishing at work of nurses, as well as to evaluate the mediating role of emotional social support on those relationships. The sample consisted of 515 nurses. The results of structural equation modeling indicated that dispositional forgiveness and emotional social support were positively related to flourishing at work. In addition, emotional social support mediated partially the relationship between dispositional forgiveness and flourishing at work. Schooling had also an impact on flourishing at work. The partial mediation model presented adequate fit indexes. The results demonstrated the relevance of emotional social support for the flourishing state at work, as well as its relation to dispositional forgiveness. The study has advanced on the explanation of some factors responsible for flourishing at work in nurses, an occupational category of great importance for the society.
\end{abstract}

Keywords: Flourishing atWork, Emotional Social Support, Dispositional Forgiveness, Well-Being at Work. 


\title{
Florecimiento en el Trabajo: Impacto del Perdón Disposicional y del Soporte Social Emocional
}

\begin{abstract}
Resumen: El florecimiento en el trabajo ha despertado interés creciente de los estudiosos en el contexto del modelo de Demandas y Recursos del Trabajo. El objetivo de este estudio fue investigar las relaciones de un recurso personal (perdón disposicional) y de un recurso de trabajo (soporte social emocional) con el florecimiento en el trabajo de enfermeros, así como evaluar el papel mediador del soporte social emocional en esas relaciones. La muestra fue compuesta por 515 trabajadores de enfermería. Los resultados del modelaje de ecuaciones estructurales señalan que el perdón disposicional y el soporte social emocional se relacionan positivamente al florecimiento en el trabajo. Además, el soporte social emocional medió parcialmente la relación entre el perdón disposicional y el florecimiento en el trabajo. La escolaridad también ha ejercido impacto sobre el florecimiento en el trabajo. El modelo de mediación parcial presentó índices de ajuste adecuados. Los resultados demostraron la relevancia del soporte social emocional para el florecimiento en el trabajo, así como su relación con el perdón disposicional. El estudio avanzó, por lo tanto, en la explicación de algunos de los factores responsables por el florecimiento en el trabajo de enfermeros, categoría de gran importancia hacia la sociedad en general.
\end{abstract}

Palabras clave: Florecimiento en el Trabajo, Soporte Social Emocional, Perdón Disposicional, Bienestar en el Trabajo.

\section{Introdução}

Atualmente as organizações vêm passando por mudanças constantes, motivadas, sobretudo, pela globalização, pelas necessidades de adaptação às novas tecnologias, pelas alterações nos valores de funcionários e clientes e pela constante exigência de cada vez maior produtividade. Tal conjuntura vem acarretando consequências nem sempre benéficas para os empregados, na medida em que ela costuma trazer prejuízos à sua saúde e a seu bem-estar laboral (Rodríguez-Carvajal, Moreno-Jiménez, Rivas-Hermosilla, Álvarez-Bejarano, \& Sanz-Vergel, 2010).

O bem-estar no trabalho vem sendo operacionalizado por meio de diferentes indicadores (Dessen, \& Paz, 2010). Um dos mais antigos e frequentes é a satisfação laboral, que consiste em uma resposta afetiva positiva ou prazerosa às atividades laborais (Locke, 1976). Outro indicador estudado com certa frequência são os afetos positivos (entusiasmo, otimismo, satisfação, orgulho, entre outros), que se associam a emoções positivas dirigidas ao contexto laboral (Ferreira, Silva, Fernandes, \& Almeida, 2008).

Mais recentemente, o florescimento no trabalho também vem sendo considerado como um indicador do bem-estar laboral e foi um dos construtos utilizados na presente investigação. $\mathrm{O}$ referido construto consiste em um estado pleno de saúde mental, no qual o indivíduo apresenta maior frequência de emoções positivas, além de demonstrar êxito psicológico e social (Keyes, 2002). Aplicado ao contexto do trabalho, ele diz respeito à prosperidade e ao desenvolvimento adquiridos no contexto laboral (Mendonça, Caetano, Ferreira, Sousa, \& Silva, 2014).

Um dos modelos que pode ser adotado na explicação do florescimento no trabalho é o de Demandas e Recursos do Trabalho (JD-R). De acordo com tal modelo, o contexto do trabalho impacta o empregado por meio de dois processos psicológicos que agem paralelamente: o primeiro, o processo de adoecimento, é provocado por demandas que exaurem a energia do empregado e levam ao desenvolvimento do estresse. O segundo, processo motivacional, advém de recursos do trabalho que estimulam o crescimento do empregado e o alcance de suas metas de trabalho (Bakker, \& Demerouti, 2007), razão pela qual contribui para o florescimento laboral. O modelo prevê ainda que os recursos pessoais também podem impactar no florescimento no trabalho (Hobfoll, Johnson, Ennis, \& Jackson, 2003).

As investigações sobre o florescimento no trabalho vêm progressivamente aumentando (Mendonça et al., 2014). No entanto, uma verificação mais 
cuidadosa dessa literatura, utilizando-se como procedimento de busca as palavras-chave: "enfermeiro/ nurse e florescimento/flourishing", demonstra que estudos sobre o florescimento no trabalho de enfermeiros ainda são inexistentes, tanto no Brasil quanto no exterior, conforme levantamento realizado em bases de dados nacionais e internacionais (IndexPsi, PePSIC, SciELO, LILACS e Banco de Teses e Dissertações da Coordenação de Aperfeiçoamento de Pessoal de Nível Superior - Capes), em julho de 2017. No entanto, o estudo dos fatores associados ao florescimento do enfermeiro reveste-se de particular relevância, na medida em que a natureza da atividade profissional desses indivíduos pode ter repercussões positivas ou negativas na saúde dos pacientes e nas próprias instituições em que eles se encontram inseridos (Gomes, Cruz, \& Cabanelas, 2009).

Justifica-se, dessa forma, a realização de estudos futuros sobre condições que propiciem o florescimento dos enfermeiros e que se mostrem capazes de ampliar os conhecimentos sobre a rede nomológica de tal construto, nessa categoria ocupacional específica. Tais estudos poderão, ainda, contribuir para o desenvolvimento de modelos de intervenção de natureza preventiva, que levem a mudanças na organização do trabalho dos enfermeiros, voltadas à promoção de seu bem-estar laboral. Fundamentando-se, portanto, nessas considerações e no modelo JD-R, este estudo teve como principal objetivo a identificação das relações de um recurso pessoal (perdão disposicional) e de um recurso do trabalho (suporte social) com o florescimento no trabalho de enfermeiros. Adicionalmente, procurou-se verificar também o impacto de características pessoais (sexo, idade e escolaridade) sobre o florescimento.

\section{O florescimento no trabalho}

$\mathrm{O}$ estudo do florescimento tem suas origens em duas abordagens tradicionalmente adotadas na investigação do bem-estar que, apesar de relativamente diferentes, se sobrepõem em alguns aspectos (Ryan \& Deci, 2001), quais sejam o bem-estar subjetivo e o bem-estar psicológico (Deci, \& Ryan, 2008). O primeiro toma como base a satisfação com a vida e a felicidade em geral (Keyes, Shmotkin, \& Ryff, 2002), enquanto o segundo sustenta-se na realização das potencialidades do ser humano (Ryan, \& Deci, 2001).

Nesse sentido, o bem-estar subjetivo associa-se à avaliação consciente que o indivíduo faz de suas experiências de vida, conforme suas próprias normas, ou seja, às reações cognitivas e afetivas sobre sua vida em geral e sobre domínios específicos da mesma (Diener, \& Suh, 1997), manifestando-se, portanto, no estado de satisfação que ele tem de si mesmo e do contexto ao seu redor (Ryan, \& Deci, 2001). Dessa forma, o bem-estar subjetivo compreende três elementos correlacionados entre si: satisfação com a vida (julgamentos de satisfação com a vida de um modo geral), afetos positivos (reações emocionais agradáveis) e afetos negativos (reações emocionais desagradáveis) (Diener, \& Suh, 1997).

O bem-estar psicológico, por sua vez, manifesta-se por meio de um processo de busca do indivíduo pela excelência, que torna possível a realização de suas habilidades (Ryff, 1995), estando assim relacionado ao funcionamento positivo (Ryff, 1989). De acordo com Ryff e Keyes (1995), o bem-estar psicológico inclui seis componentes: autoaceitação (julgamentos positivos de si mesmo e de eventos passados); crescimento pessoal (percepção constante de desenvolvimento das habilidades); propósito na vida (convicção de que a vida é significativa); relações positivas (manutenção de relacionamentos saudáveis); domínio ambiental (aptidão para conduzir a vida e o contexto); autonomia (senso de independência). Tais elementos podem ser considerados como componentes-chave do bem-estar psicológico, pois representam o aspecto saudável e pleno do funcionamento humano (Ryff, \& Singer, 2008).

Com o intuito de unir essas duas correntes do bem-estar, Keyes (2006) introduz o termo florescimento, para designar um estado de saúde mental completo, caracterizado pela ausência de psicopatologias e pela abundância de bem-estar emocional, psicológico e social. De modo semelhante, o florescimento pode ser entendido como um estado que envolve um alto nível de bem-estar psicológico, identificado nas situações que o indivíduo se sente bem e produz eficientemente (Huppert, \& So, 2009).

Segundo esta perspectiva, o florescimento é constituído por um conjunto de características, sendo que três delas são centrais (emoções positivas, engajamento/interesse, significado/propósito) e seis, complementares (autoestima, otimismo, resiliência, vitalidade, autodeterminação e relacionamentos positivos) (Huppert, \& So, 2009). O florescimento pode ser caracterizado, portanto, por uma vida prazerosa, que integra o bem-estar subjetivo (satisfação 
com a vida, nível elevado de afetos positivos e nível reduzido de afetos negativos), a saúde e o funcionamento positivo (Mendonça et al., 2014).

Aplicado ao contexto do trabalho, o florescimento caracteriza-se pelo ajustamento psicológico do trabalhador ao contexto laboral no qual está inserido (Carver, \& Connor-Smith, 2010), manifesto em sentimentos e emoções positivas e em bom funcionamento psicológico e social (Dutton, Roberts, \& Bednar, 2010). Em suma, o florescimento no trabalho pode ser considerado como a integração do bem-estar subjetivo e psicológico no contexto laboral.

De acordo com o Modelo de Demandas e Recursos do Trabalho, o bem-estar no trabalho e todas as suas manifestações, entre as quais se inclui o florescimento no trabalho, sofre a influência de demandas e recursos do trabalho (Demerouti, Bakker, Nachreiner, \& Schaufeli, 2001). As demandas associam-se a características físicas, psicológicas, sociais ou organizacionais do trabalho que exigem grande esforço físico ou mental, razão pela qual costumam provocar danos fisiológicos ou psicológicos no empregado que podem até levá-lo à exaustão (Demerouti et al., 2001), ao estresse e ao burnout (Bakker, \& Demerouti, 2007). Dentre os exemplos de demandas do trabalho estão a sobrecarga, a pressão do tempo e os ambientes físicos difíceis (Crawford, LePine, \& Rich, 2010). As demandas do trabalho impactam assim negativamente o florescimento no trabalho.

Os recursos do trabalho, por sua vez, dizem respeito aos aspectos físicos, psicológicos, sociais ou organizacionais do trabalho que protegem a saúde do trabalhador, ao lhe ajudarem a alcançar suas metas de trabalho e estimularem seu desenvolvimento e crescimento pessoal, bem como diminuírem o impacto das demandas e os custos fisiológicos e psicológicos associados às tarefas (Demerouti et al., 2001). Além de serem considerados um direito do trabalhador, os recursos do trabalho são, portanto, de fundamental importância para que o mesmo possa reagir às elevadas exigências do trabalho (Bakker, 2011).

Dentre os exemplos de recursos do trabalho estão o controle do tempo, o suporte do líder, os relacionamentos confiáveis com os colegas (Bakker, Demerouti, \& Schaufeli, 2003), o feedback e o suporte social (Crawford et al., 2010). Os recursos de trabalho relacionam-se, portanto, positivamente ao florescimento no trabalho, em função de desempenharem um papel motivacional intrínseco, associado à satis- fação das necessidades humanas básicas, e um papel motivacional extrínseco, relacionado ao desenvolvimento pessoal e ao alcance de metas (Schaufeli, \& Bakker, 2004).

De acordo com o modelo JD-R, os recursos pessoais também impactam positivamente no florescimento no trabalho, e se caracterizam como aspectos positivos pessoais que habilitam o indivíduo a controlar e a influenciar seu ambiente com sucesso (Hobfoll et al., 2003). Por essa razão, tais recursos contribuem para o ajustamento satisfatório do indivíduo às situações cotidianas (Hobfoll, 2002). Dentre os exemplos de recursos pessoais estão a autoeficácia, a autoestima de base organizacional e o otimismo (Xanthopoulou, Bakker, Demerouti, \& Schaufeli, 2007). Em síntese, tanto os recursos do trabalho quanto os recursos pessoais, juntos ou separados, são fundamentais para o florescimento no trabalho (Hobfoll, 2002).

O presente trabalho baseou-se apenas no lado positivo do modelo JD-R, isto é, abordou apenas as relações de um recurso pessoal e de um recurso do trabalho com o florescimento. De modo mais específico, foram analisadas as relações do perdão disposicional (recurso pessoal) e do suporte social (recurso do trabalho) com o florescimento no trabalho de enfermeiros.

\section{O perdão disposicional e o florescimento no trabalho}

O perdão disposicional ou intrapessoal caracteriza-se como uma firme tendência do indivíduo para perdoar o outro em qualquer tempo ou situação (Berry, Worthington, Parrott III, O'Connor, \& Wade, 2001). Ele consiste, portanto, em um traço psicológico permanente, que varia entre os indivíduos, ou seja, em uma força de caráter manifesta na capacidade intrapsíquica do indivíduo de não se deixar esmorecer com os danos causados pelo outro (Gouveia, et al., 2015).

No contexto do modelo JD-R, os recursos pessoais dizem respeito às tendências psicológicas que ajudam o indivíduo a manter um senso de controle sobre o ambiente (Van den Heuvel, 2013), facilitando, assim, sua adaptação às modificações e situações da vida (Hobfoll, 2002), ao possibilitarem o enfrentamento de circunstâncias adversas (Van den Heuvel, 2013). Os recursos pessoais facilitam, desse modo, o alcance de metas na presença de acontecimentos difíceis, estressantes ou ambíguos (Xanthoupoulou et al., 2007). O perdão disposicional pode ser visto, portanto, 
como uma característica ou recurso pessoal (Berry, Worthington, O'Connor, Parrott III, \& Wade, 2005) que contribui para a saúde física e mental (Reed, \& Enright, 2006), ao promover bons resultados tanto para os indivíduos, quanto para as relações no contexto social (Berry et al., 2005).

A relação direta entre o perdão e a saúde geral pode ser também explicada pelo modelo conceitual proposto por Worthington Jr, Berry e Parrott III (2001). De acordo com os autores, quando os indivíduos vivenciam a falta do perdão, por meio da ruminação e de emoções de ressentimento, amargura, ódio, raiva residual, medo (Worthington Jr et al., 2001), retaliação e vingança (Worthington Jr, \& Wade, 1999), eles podem apresentar problemas de saúde mental (Toussaint, \& Webb, 2005).

Contudo, quando as emoções negativas sofrem a influência do perdão, elas se transformam em emoções fortes e positivas, baseadas no amor (Worthington Jr et al., 2001), sendo que tais mudanças envolvem uma variedade de processos fisiológicos (Toussain,t \& Webb, 2005). Corroborando tais argumentos, estudos empíricos têm evidenciado que o perdão disposicional apresenta correlações positivas com o bem-estar (Toussaint, \& Friedman, 2008), o bem-estar subjetivo, o bem-estar psicológico (Lawler-Row \& Piferi, 2006), a saúde mental (Al-Sabeelah, Alraggad, \& Ameerh, 2014) e os afetos positivos (Thompson et al., 2005).

Seria assim de se esperar que os indivíduos com maiores níveis de perdão disposicional apresentassem maior facilidade de adaptação ao contexto laboral como forma de atingir suas metas, florescendo assim, no trabalho. Fundamentando-se, portanto, no modelo JD-R, bem como nos achados empíricos apresentados, formulou-se a seguinte hipótese:

H1 - O perdão disposicional relaciona-se positivamente ao florescimento no trabalho.

\section{O suporte social emocional e o florescimento no trabalho}

O suporte social diz respeito à rede de relacionamentos e transações sociais que cercam o indivíduo e possibilitam seu enfrentamento adaptativo, em casos de dificuldades (Sarason, \& Duck, 2001), sendo, assim, inerente à natureza da rede social que cerca o indivíduo (Andrade, Hoch, Vieira, \& Rodrigues, 2012). No contexto do trabalho, tal fenômeno diz respeito ao modo pelo qual os indivíduos percebem a existência e a disponibilidade de apoio social, bem como à qualidade do relacionamento interpessoal com a chefia e com os colegas (Tamayo, \& Tróccoli, 2002). Ele favorece, portanto, a interação e o apoio recíproco entre colegas de trabalho, colaborando para o aperfeiçoamento do ambiente de trabalho, para os relacionamentos entre as pessoas e a organização (Andrade et al., 2012), e para a expressão e realização pessoais (Paschoal, Torres, \& Porto, 2010).

De acordo com Gomide Junior, Guimarães e Damásio (2004), o suporte social no trabalho pode ser classificado em emocional, instrumental e informacional. $\mathrm{O}$ suporte emocional refere-se às ações e palavras ditas ao outro (ser empático e confiável, ser conselheiro e ouvinte), que são por ele entendidas como atos de afeição, cuidado e atenção. O suporte instrumental envolve o auxílio material ou prático providos a alguém (suprimento financeiro, atenção e apoio às atividades diárias). O suporte informacional, por fim, compreende o recebimento de informações que direcionam e orientam o indivíduo na tomada de decisões para a resolução de problemas. No presente trabalho foi adotado apenas o suporte social emocional, por ser ele o primeiro a surgir nas relações interpessoais (Rodriguez, \& Cohen, 1998), envolvendo a manifestação de empatia, cuidado, segurança e confiança que favorecem a expressão e a liberação das emoções (Cohen, 2004).

De acordo com o modelo JD-R, o suporte social pode ser caracterizado como um recurso do trabalho que se reveste de qualidades motivacionais intrínsecas e extrínsecas (Bakker et al., 2003). Seria assim de se esperar que o fato de os empregados usufruírem de suporte e incentivo de seus colegas de trabalho e supervisores desencadeasse um processo de motivação intrínseco aliado à satisfação das necessidades de pertencimento e um processo de motivação extrínseco, evidenciado pela aplicação de esforços para o alcance das metas de trabalho.

Em apoio a tais argumentos, o suporte social tem apresentado relações positivas sistemáticas com diferentes manifestações de bem-estar laboral, na medida em que sua falta constitui-se em uma fonte de estresse, que aumenta os níveis de burnout (Leite, 2007), enquanto sua presença contribui para o bem-estar e para a saúde física e mental dos trabalhadores (Tamayo, \& Tróccoli, 2002; Van Yperen, 1998). Nesse sentido, os estudos empíricos têm apontado que ele apresenta associações positivas com diferentes resultados positivos individuais advin- 
dos do contexto do trabalho, como, por exemplo, o envolvimento no trabalho (Ali, \& Panatik, 2013), o engajamento no trabalho (Burke, Moodie, Dolan, \& Fiksenbaum, 2012; Schaufeli, \& Bakker, 2004), a dedicação ao trabalho (Bakker et al., 2003) e o comprometimento organizacional (Bakker et al., 2003; Humphrey, Nahrgang, \& Morgeson, 2007), a satisfação no trabalho (Ali, \& Panatik, 2013; Ducharme, \& Martin, 2000; Hombrados-Mendieta, \& Cosano-Rivas, 2013; Humphrey et al., 2007) e os afetos positivos dirigidos ao trabalho (Paschoal et al., 2010). Fundamentando-se, portanto, no modelo JD-R e nos estudos revisados, formulou-se a seguinte hipótese:

$\mathrm{H} 2$ - O suporte social emocional relaciona-se positivamente ao florescimento no trabalho.

\section{O suporte social emocional como mediador das relações entre o perdão disposicional e o florescimento no trabalho}

Segundo o modelo de Worthington et al. (2001), o perdão apresenta também efeitos positivos indiretos na saúde mental, por meio de determinadas variáveis mediadoras como, por exemplo, o suporte social (Temoshok, \& Chandra, 2000; Worthington Jr. et al., 2001). Nesse sentido, uma pessoa que é capaz de perdoar os outros e de abrir mão dos sentimentos improdutivos de raiva, amargura, ressentimento ou decepção, provavelmente será capaz de buscar e receber mais facilmente e eficazmente o suporte social emocional proveniente dos demais membros de sua rede de relações sociais (Temoshok, \& Wald, 2005). Postula-se, assim, que o perdão pode levar a uma rede social mais ampla e satisfatória de apoio emocional (Worthington Jr. \& Scherer, 2004) e promover o reparo de relacionamentos (Worthington Jr, \& Drinkard, 2000), o que resulta em uma variedade de resultados saudáveis no que diz respeito à saúde (Worthington Jr et al., 2001). Em suma, o perdão disposicional relaciona-se positivamente ao suporte social emocional que, por sua vez, associa-se positivamente à saúde mental.

Apoiando-se em tais argumentos, estudos empíricos têm apontado o suporte social como mediador das relações entre o perdão e o bem-estar subjetivo, o perdão e o bem-estar psicológico, o perdão e a saúde (Berry et al., 2005; Lawler-Row, \& Piferi, 2006; Webb, Hirsch, Visser, \& Brewer, 2013) e o perdão e a satisfação com a vida (Zhu, 2015). Seria assim de se esperar que o suporte social atuasse como um mediador das relações entre o perdão disposicional e o florescimento no trabalho. Tomando-se por base o modelo conceitual proposto por Worthington Jr et al. (2001) e os estudos revisados, foi então formulada a seguinte hipótese:

H3 - O suporte social emocional medeia a relação do perdão disposicional com o florescimento no trabalho.

\section{O florescimento no trabalho e as características pessoais}

O estado de florescimento resulta da interação de características pessoais, habilidades para o desenvolvimento das atividades e contexto (Salanova, Bakker, \& Llorens, 2006). Entretanto, não há clareza sobre o impacto de características pessoais, tais como sexo, idade e escolaridade, sobre as experiências de florescimento.

Observa-se o impacto de características sociodemográficas e laborais em outros estados de bem-estar vivenciados no trabalho (Vazquez, Freitas, Cyrre, Hutz, \& Schaufeli, 2018; Vazquez, Magnan, Pacico, Hutz, \& Schaufeli, 2015; Zacher, Jimmieson, \& Bordia, 2014). Por exemplo, foi observado que entre profissionais brasileiros, o aumento da idade está associado ao aumento dos índices de engajamento (Vazquez et al., 2015). Em uma amostra de profissionais australianos, foi observado que a idade apresentou uma relação curvilínea com a satisfação com o trabalho e a exaustão emocional. Desta forma, profissionais na faixa etária dos 20 aos 40 anos apresentaram índices de satisfação com o trabalho mais baixos e níveis de exaustão emocional mais altos, em comparação aos profissionais mais jovens (idade menor de 20 anos) e mais velhos (idade superior a 40 anos) (Zacher et al., 2014).

Em relação ao sexo, estudo realizado com profissionais brasileiros, foi observado que as mulheres apresentavam médias significativamente superiores aos homens na dimensão trabalhar excessivamente do workaholism. Além disso, profissionais com maior escolaridade obtiveram índices significativamente mais altos do que os outros participantes nas dimensões trabalhar excessivamente e trabalhar compulsivamente do workaholism (Vazquez et al., 2018). Considerando que o florescimento se caracteriza como um estado de bem-estar no trabalho, a quarta hipótese deste estudo propôs:

H4 - As características sexo, idade e escolaridade apresentarão uma relação positiva de baixa magnitude com o florescimento no trabalho. 
Considerando-se, portanto, as diferentes hipóteses formuladas, a pesquisa objetivou testar um modelo de mediação. Tal modelo encontra-se na Figura 1.

\section{Método}

\section{Participantes}

Para inclusão no estudo foi utilizado como critério o fato de o participante estar trabalhando na área de enfermagem e possuir pelo menos um ano de experiência em sua respectiva função, visto que o objetivo foi analisar o florescimento nessa categoria específica. A amostra contou com 515 trabalhadores de enfermagem (enfermeiros, técnicos e auxiliares) de ambos os sexos, provenientes do estado do Rio de Janeiro.

Dentre os respondentes, a maioria foi do sexo feminino $(72,2 \%)$, com idades variando de 18 a 54 anos $(M=30,20 ; D P=9,20)$. Quanto ao estado civil, a maior parte distribuiu-se entre solteiros $(61,0 \%)$ e casados (34,2\%). No que diz respeito à escolaridade, a representatividade maior foi de pessoas com o ensino médio completo $(48,9 \%)$ ou com o ensino superior incompleto $(43,5 \%)$. Com relação à categoria profissional, a maioria foi composta por técnicos em enfermagem (75,5\%), seguida pelos auxiliares (16,7\%). Quanto à faixa salarial, prevaleceram os participantes que recebiam entre um a três salários mínimos $(89,1 \%)$. No tocante ao setor organizacional, a maioria trabalhava no setor privado $(58,8 \%)$. Em relação ao turno de trabalho, a maioria trabalhava no turno diurno $(65,4 \%)$ ou em escala $(22,7 \%)$. Sobre a unidade de trabalho, a maior parte da amostra distribuiu-se em enfermaria (35,7\%), emergência (23,5\%) e ambulatório (22,3\%). No que tange ao tempo de atuação na categoria profissional, a variação foi de 1 a 30 anos $(M=4,51 ; D P=5,26)$, enquanto o tempo no emprego atual variou de 1 a 29 anos $(M=2,79 ; D P=3,98)$.
Com referência ao número de horas trabalhadas por semana, o tempo variou de 4 a 60 horas $(M=34,58$; $D P=10,75)$.

\section{Instrumentos}

Para a mensuração do perdão disposicional utilizou-se a Escala de Traço de Perdoabilidade (TFS), desenvolvida por Berry et al. (2005) e adaptada para amostras brasileiras no estudo de Chaves (2017). Esta escala é de natureza unifatorial e composta por sete itens, a serem respondidos em escala Likert de cinco pontos, variando de: discordo fortemente (1) a concordo fortemente (5). Exemplo de item: "Eu sou uma pessoa que perdoa". No estudo de evidências de validade, a escala obteve Alfa de Cronbach igual a 0,70 e, na presente investigação, este índice foi de 0,67. Cumpre ressaltar que na análise fatorial confirmatória da Escala de Traço de Perdoabilidade, o item 1 "As pessoas próximas a mim provavelmente acham que eu guardo rancor por muito tempo" não foi significativo e, por este motivo, optou-se pela retirada de tal item, o que elevou o alfa de 0,64 inicialmente obtido para 0,67 . No presente estudo a escala apresentou ainda índices de ajuste satisfatórios $\left(\chi^{2}(8)=20,42\right.$; $\mathrm{p}<0,001) ; \mathrm{CFI}=0,99 ;$ TLI $=0,98 ;$ RMSEA $=0,05$ $(90 \%$ I.C. $=0,03-0,08)$.

O suporte social emocional foi avaliado pela subescala destinada a esse fim na Escala de Percepção de Suporte Social no Trabalho (EPSST), desenvolvida originalmente no Brasil por Gomide Junior et al. (2004). A subescala compõe-se de seis itens, a serem respondidos em escalas tipo Likert de cinco pontos, variando de discordo fortemente (1) a concordo fortemente (5). Exemplo de item: "Na empresa onde eu trabalho, pode-se confiar nas pessoas". No estudo de validação original, o índice de consistência interna desse fator, calculado pelo Alfa de Cronbach, foi igual

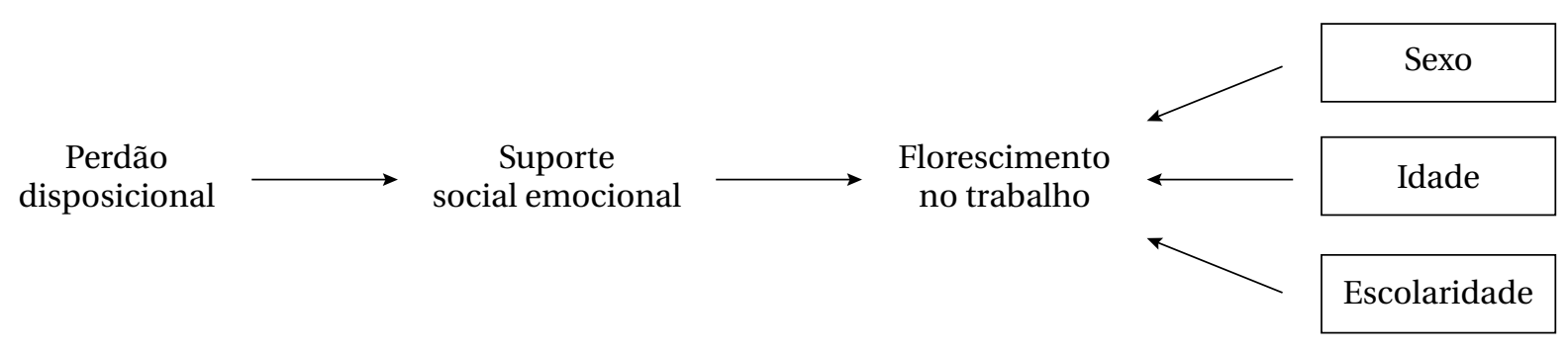

Figura 1

Modelo hipotético testado. 
a 0,83 . No presente estudo, esse coeficiente foi igual a 0,75 , com os índices de ajuste mostrando-se adequa$\operatorname{dos}\left(\chi^{2}(8)=39,89 ; \mathrm{p}<0,001\right) ; \mathrm{CFI}=0,98 ; \mathrm{TLI}=0,96$; RMSEA $=0,09$ (90\% I.C. $=0,06-0,12)$.

O florescimento no trabalho foi avaliado pela Escala de Florescimento no Trabalho (EFLOT), desenvolvida por Diener et al. (2010) e adaptada para amostras brasileiras por Mendonça et al. (2014). A escala é unifatorial e compõe-se de oito itens, a serem respondidos em escalas tipo Likert de sete pontos, variando de discordo completamente (1) a concordo completamente (7). Exemplo de item: "Em meu trabalho as pessoas me respeitam”. No estudo de validação inicial da escala, o índice de consistência interna desse fator, calculado pelo Alfa de Cronbach, foi igual a 0,91 e, no presente estudo, ele foi de 0,87 . Os índices de ajuste da escala de florescimento, na presente amostra, foram satisfatórios $\left(\chi^{2}(18)=388,15 ; \mathrm{p}<0,001\right)$; CFI $=0,95$; $\mathrm{TLI}=0,93 ; \mathrm{RMSEA}=0,20(90 \%$ I.C. $=0,18-0,22)$.

Foi aplicado ainda um Questionário Sociodemográfico e Laboral para investigar as características dos participantes, como sexo, idade e escolaridade. O questionário foi composto por oito itens, referentes à descrição de informações relativas a dados sociodemográficos dos participantes, bem como de sua situação laboral.

\section{Procedimento de coleta de dados}

Em atendimento aos critérios da Resolução CNS $n^{\circ} 466$, de 12 de dezembro de 2012, que regula a Ética na Pesquisa com Seres Humanos, a pesquisa foi inicialmente submetida ao Comitê de Ética em Pesquisa da instituição dos autores e aprovada com o $\mathrm{n}^{\circ}$ 1.843.114. Após a sua aprovação, a coleta de dados foi realizada por meio de formulário impresso.

Os questionários foram entregues e recolhidos logo após o seu preenchimento, sem a mediação de qualquer outra pessoa das organizações em que os participantes trabalhavam. Para a participação na pesquisa houve concordância prévia, mediante $o$ preenchimento do Termo de Consentimento Livre e Esclarecido (TCLE). A participação foi voluntária e o anonimato do indivíduo e da organização foram preservados.

\section{Procedimento de análise de dados}

Com o intuito de verificar a validade discriminante das variáveis compreendidas pelo estudo, foi feito o cálculo das Variâncias Médias Extraídas (VME), que deve ser maior que as correlações ao quadrado de cada uma delas, como um indicador de ajuste do modelo (Fornell, \& Larcker, 1981). Em seguida, foi realizada a Modelagem de Equações Estruturais, usando-se o software Mplus versão 6.12, a fim de investigar o papel mediador do suporte social emocional nas relações do perdão disposicional com o florescimento no trabalho dos enfermeiros.

O papel mediador do suporte social emocional foi testado por meio de três passos. Inicialmente, foi avaliado se o perdão disposicional estava relacionado ao florescimento no trabalho. Posteriormente, a relação do perdão disposicional com o florescimento no trabalho, na presença do suporte social emocional, foi investigada, assim como as relações do suporte social emocional com o florescimento no trabalho. Após isso, foi investigado se o suporte social emocional mediava a relação entre o perdão disposicional e o florescimento no trabalho. Por fim, para verificar os efeitos de mediação, foi calculado o intervalo de confiança de $95 \%$ dos efeitos de mediação, utilizando-se a técnica de bootstrapping (1.000 reamostragens). Nos três passos foram controlados os efeitos para sexo, idade e escolaridade dos participantes.

Os parâmetros foram estimados por meio da Weighted Least Squares Means and Variance Adjusted (WLSMV), pois este método é recomendado para dados ordinais sem distribuição normal. Apoiando-se nas recomendações de Byrne (2001), foram analisados os seguintes índices de ajuste: qui-quadrado (testa a probabilidade do modelo teórico se ajustar aos dados, sendo que quanto maior o valor do $\chi^{2}$, pior o ajustamento); Root Mean Square Error of Approximation (RMSEA) - deve se situar abaixo de 0,08 , aceitando-se valores até 0,10; Tucker-Lewis Index (TLI) - são considerados ajustados modelos com valores acima de 0,90; Comparative fit index (CFI) - são considerados ajustados valores acima de 0,90.

\section{Resultados}

Inicialmente, foram calculadas as correlações entre as variáveis do modelo, os quadrados dessas correlações e as VMEs (Tabela 1). Os resultados mostraram que todas as variáveis apresentaram correlações significativas e que os VMEs das variáveis foram maiores do que suas correlações ao quadrado, demonstrando que houve validade discriminante entre elas. 
No que diz respeito ao teste das hipóteses do estudo foi, primeiramente, testado o modelo de efeitos diretos da variável perdão sobre o florescimento. Os resultados indicaram que o efeito foi significativo (perdão e florescimento, $\beta=0,37 ; p<0,001$ ), ou seja, o perdão relacionou-se positivamente ao florescimento no trabalho, o que confirmou a hipótese 1. Além disso, foi observado que a escolaridade esteve positivamente relacionada às experiências de florescimento no trabalho ( $\beta=0,19 ; p<0,001$ ), sendo que quanto maior a especialização técnica dos profissionais, maiores os índices de florescimento no trabalho, como proposto na hipótese 4. As características de sexo e idade não apresentaram um impacto significativo sobre os índices de florescimento no trabalho, diferente do esperado na hipótese 4.

Um segundo modelo foi testado para verificar o efeito direto do perdão sobre o florescimento, na presença da variável suporte social emocional. Os resultados se mantiveram significativos (perdão e florescimento, $\beta=0,32 ; p<0,001$ ) e demonstraram a relação entre o suporte social emocional e florescimento ( $\beta=0,24 ; p<0,001)$. Confirmou-se, assim, a hipótese 2 , a qual previa que o suporte social emocional se relacionaria positivamente ao florescimento no trabalho.
A escolaridade, entre as características sociodemográficas investigadas, permaneceu apresentando um impacto positivo sobre os índices de florescimento $\left(\chi^{2}=0,19 ; p<0,001\right)$, corroborando parcialmente a hipótese 4. As outras variáveis sociodemográficas avaliadas no modelo (sexo e idade) não impactaram sobre o florescimento, refutando parcialmente o proposto na hipótese 4 .

No terceiro modelo, foi testado o efeito de mediação do suporte social emocional. Constatou-se a existência de uma mediação parcial do suporte social emocional na relação entre o perdão e o florescimento (Tabela 2), que confirmou a hipótese 3. A análise do percentual do efeito indireto específico demonstrou que o suporte social emocional mediou $18,75 \%$ da relação entre o perdão e o florescimento. A escolaridade permaneceu sendo uma característica laboral que impacta os índices de florescimento no trabalho, confirmando parcialmente a hipótese 4. Enquanto as características sociodemográficas sexo e idade não contribuíram para a explicação do florescimento, ao contrário do proposto na hipótese 4 . O modelo mediacional final, que pode ser observado na Figura 2, apresentou bons índices de ajuste: $\chi^{2}=711,47$ (239), CFI $=0,95$; TLI $=0,95 \mathrm{e}$ RMSEA $=0,06$ (95\% I.C., 0,06 a 0,07).

Tabela 1

Resultado da análise discriminante das variáveis.

\begin{tabular}{lcccc}
\hline & VME & SSEMOC & PERDÃO & FLORESC \\
\hline SSEMOC & 0,40 & - & 0,06 & 0,12 \\
PERDÃO & 0,37 & 0,25 & - & 0,11 \\
FLORESC & 0,57 & 0,34 & 0,33 & - \\
\hline
\end{tabular}

Nota: Na diagonal inferior são apresentadas as correlações entre as variáveis latentes, estimadas por meio de equações estruturais. Na diagonal superior são apresentados os coeficientes de determinação (isto é, o quadrado da correlação). Suporte Social Emocional (SSEMOC); Florescimento (FLORESC). Todos os valores foram significativos $(p<0,001)$.

Tabela 2

Efeito de mediação do Suporte Social Emocional na relação entre Florescimento e Perdão (N = 515).

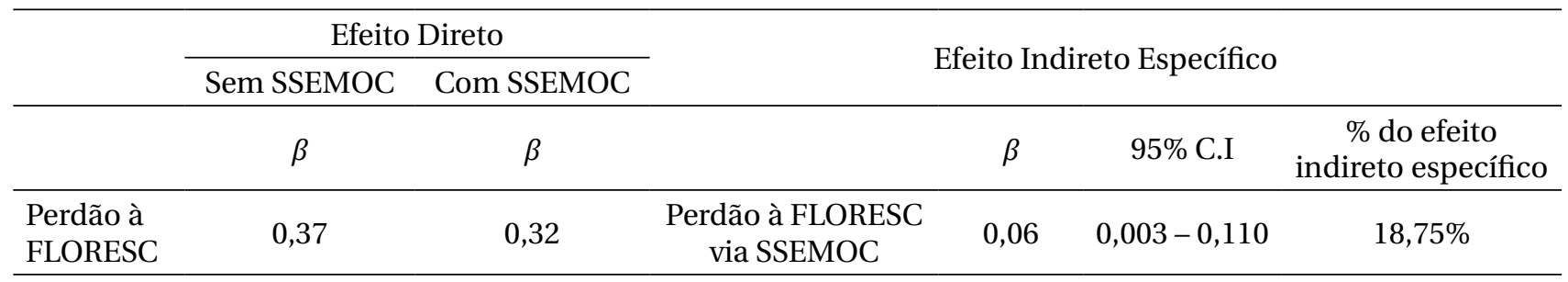

SSEMOC: Suporte Social Emocional; FLORESC: Florescimento.

Todos os efeitos foram significativos a nível $p<0,001$. 


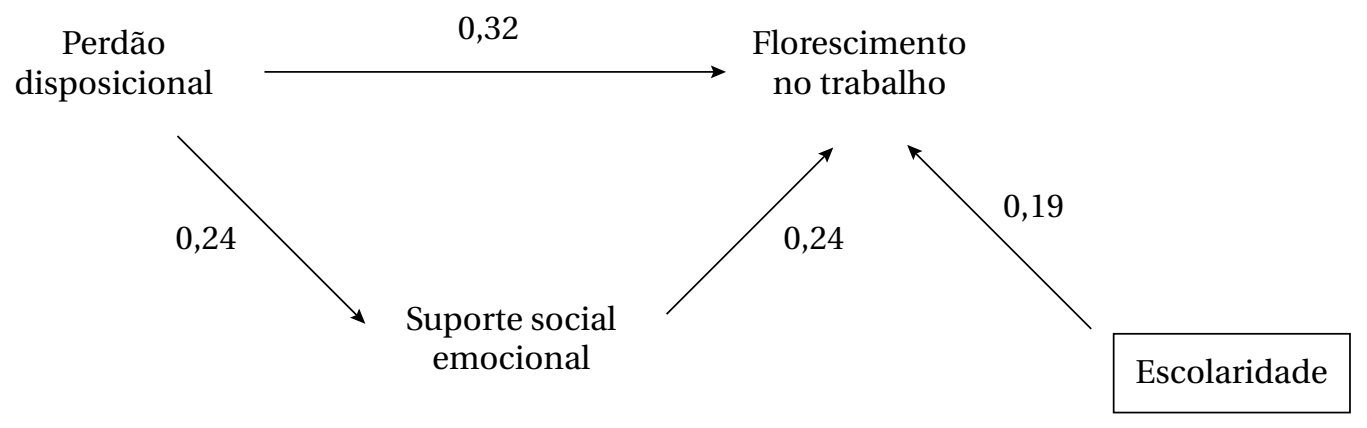

Nota: A figura é uma representação gráfica do modelo. O efeito do perdão disposicional sobre o florescimento no trabalho é o efeito direto $\left(c^{\prime}\right)$, após o controle dos efeitos de mediação. Todos os efeitos foram significativos a nível $p<0,001$.

Figura 2

Modelo mediacional final com os parâmetros padronizados.

\section{Discussão}

O presente estudo teve como objetivo geral investigar os efeitos de um recurso pessoal (perdão disposicional) e de um recurso do trabalho (suporte social) sobre o florescimento no trabalho de enfermeiros. Os resultados obtidos por meio da modelagem de equações estruturais evidenciaram que a Hipótese 1 , a qual previa que o perdão disposicional iria predizer positivamente o florescimento no trabalho dos enfermeiros, foi confirmada. Evidenciou-se, dessa forma, que quanto maiores os níveis de perdão disposicional, maiores são os índices de florescimento no trabalho dos enfermeiros. Esses achados corroboram pesquisas anteriores que também constataram que o perdão disposicional apresentou correlações positivas com o bem-estar (Toussaint, \& Friedman, 2008), o bem-estar subjetivo, o bem-estar psicológico (Lawler-Row, \& Piferi, 2006), a saúde (Worthington Jr, Witvliet, Pietrini, \& Miller, 2007), a saúde mental (Al-Sabeelah et al., 2014), os afetos positivos e a satisfação com a vida (Thompson et al., 2005).

O perdão disposicional está relacionado à tendência natural do indivíduo em perdoar um ofensor, independentemente do tempo ou contexto (Berry et al., 2001). Considerando-se que os profissionais de enfermagem realizam suas funções a partir de um trabalho coletivo caracterizado por uma diversidade de pessoas, em que a cooperação e a comunicação são elementos fundamentais para uma boa relação no ambiente laboral (Damasceno, Campos, Falco, Souza, \& Penna, 2009), é possível supor que os profissionais que possuem uma disposição contínua para perdoar (Roberts, 1995) não se deixem abater pelos prejuízos causados pelo outro
(Gouveia et al., 2015), o que se reverteria em resultados positivos para sua saúde física e mental, ou seja, para seu florescimento no trabalho. Tal resultado corrobora ainda com evidências empíricas em apoio ao Modelo de Demandas e Recursos do Trabalho (Xanthoupoulou et al., 2007), ao demonstrar que o perdão disposicional, enquanto um recurso pessoal, contribui para o florescimento no trabalho.

O suporte social emocional também se relacionou positivamente ao florescimento no trabalho, confirmando, assim, a Hipótese 2. Constatou-se, assim, que quanto maiores os níveis de suporte social emocional, maiores são os índices de florescimento no trabalho dos enfermeiros. Em outras palavras, o fato de os enfermeiros perceberem uma rede de relacionamentos positivos que lhes dá suporte emocional em seu local de trabalho contribui para seu bem-estar subjetivo e psicológico, isto é, para seu florescimento no contexto laboral.

Tal resultado confirma dados obtidos em outra investigação, na qual também se verificou que o suporte social emocional exerceu influência positiva sobre o bem-estar (Estivalete, Andrade, Faller, Stefanan, \& Souza, 2013). Outros estudos também observaram que o suporte social geral se relacionou positivamente com a satisfação no trabalho (Ali, \& Panatik, 2013; De Jonge, \& Schaufeli, 1998; Hombrados-Mendieta, \& Cosano-Rivas, 2013), o bem-estar subjetivo (Burke et al., 2012) e a dedicação ao trabalho (Bakker et al., 2003).

O resultado ora encontrado contribui ainda com evidências empíricas em apoio ao Modelo de Demandas e Recursos do Trabalho (Bakker, \& Demerouti, 2007), ao reunirem provas adicionais sobre o fato de 
que os recursos do trabalho contribuem para o florescimento no trabalho. Em outras palavras, o suporte social emocional pode ser considerado como um recurso (Crawford et al., 2010) que se relaciona ao florescimento, por exercer um papel motivacional intrínseco, aliado à satisfação das necessidades humanas básicas, e um papel motivacional extrínseco, aliado ao desenvolvimento pessoal e ao alcance de metas (Schaufeli, \& Bakker, 2004).

Foi constatado, ainda, que o suporte social emocional mediou parcialmente a relação entre o perdão disposicional e o florescimento no trabalho, o que confirmou a Hipótese 3. Verificou-se, assim, que maiores índices de perdão disposicional levam ao aumento do suporte social emocional, o qual, por sua vez, contribui para o aumento do florescimento no trabalho dos enfermeiros.

Nesse sentido, uma pessoa que é capaz de perdoar os outros e de abrir mão dos sentimentos improdutivos de raiva, amargura, ressentimento ou decepção, provavelmente será capaz de buscar e receber mais fácil e eficazmente o suporte social proveniente dos demais membros de sua rede de relações sociais (Temoshok, \& Wald, 2005). Em outras palavras, o perdão pode levar a uma rede social mais ampla e satisfatória de apoio emocional (Worthington Jr, \& Scherer, 2004) e promover a restauração de relacionamentos (Worthington Jr, \& Drinkard, 2000), o que ocasiona uma variedade de resultados saudáveis no que diz respeito à saúde (Worthington Jr et al., 2001). Dessa forma, os enfermeiros que perdoam os outros e ignoram os sentimentos negativos conseguem aumentar mais facilmente a sua rede de suporte social, o que possibilita o seu florescimento.

Este resultado converge com os achados de outros estudos nos quais o suporte social atuou como mediador das relações entre o perdão e o bem-estar subjetivo, o bem-estar psicológico, a saúde (Berry et al., 2005; Lawler-Row, \& Piferi, 2006; Webb et al., 2013) e a satisfação com a vida (Zhu, 2015). O dado presentemente obtido contribui ainda com evidências empíricas em apoio ao modelo teórico de Worthington Jr et al. (2001), segundo o qual a associação particular do perdão com a saúde se dá por meio de mediadores distintos, entre os quais se encontra o suporte social.

A escolaridade foi a única característica sociodemográfica e laboral que apresentou impacto sobre os índices de florescimento no trabalho. A relação positiva entre a escolaridade e o florescimento sugere que profissionais de enfermagem com maior escolaridade vivenciam maiores níveis de florescimento no trabalho. A relação positiva entre a escolaridade e o florescimento pode ser devido aos profissionais com maior escolaridade ocuparem cargos com maior autonomia e possibilidade de decisão no trabalho. A autonomia e decisão no trabalho podem ser compreendidas como recursos laborais, os quais estão associados ao processo motivacional no trabalho, que, por sua vez, pode promover estados de bem-estar no trabalho (Bakker, \& Demerouti, 2007). Já as demandas de profissionais de enfermagem com menor especialização técnica geralmente envolvem altos índices de demandas físicas e operacionais. $\mathrm{O}$ excesso de demandas está associado ao desenvolvimento do processo de adoecimento e a menores índices de bem-estar no trabalho (Bakker, \& Demerouti, 2007).

Cumpre ressaltar, porém, as limitações do presente estudo. A primeira está relacionada ao fato de que toda a amostra se concentrou em um único estado do Brasil, o Rio de Janeiro, o que pode dificultar a generalização dos resultados para os demais estados brasileiros. Outra limitação diz respeito ao instrumento utilizado para a medida do perdão, que foi adaptado para o contexto brasileiro com alfa razoável. Entretanto, no presente estudo, o alfa do instrumento para avaliar o perdão ficou abaixo do esperado. A natureza transversal do estudo também constitui uma limitação, por impossibilitar a inferência de relações de causalidade entre as variáveis.

No que diz respeito à realização de estudos futuros, seria interessante que investigações sobre essa temática fossem conduzidas em outras regiões brasileiras. Sugere-se, também, a realização de estudos longitudinais, que levem à obtenção de maior compreensão acerca das relações entre as variáveis da presente investigação. Por fim, sugere-se que pesquisas futuras se dediquem à investigação de outras variáveis preditoras do florescimento no trabalho de enfermeiros, como forma de contribuir para o aprofundamento da rede nomológica do referido construto.

Independentemente dessas limitações, a presente pesquisa reveste-se de relevância teórica e prática, em função de ter proposto e testado um modelo ainda não explorado anteriormente. Além disso, avançou na explicação de alguns dos fatores responsáveis pelo florescimento no trabalho de enfermeiros, categoria profissional de grande relevância para a sociedade em geral. 


\section{Conclusão}

Este estudo procurou elaborar o teste de um modelo sobre as relações diretas do perdão disposicional e do suporte social emocional com o florescimento no trabalho dos enfermeiros, bem como sobre o papel mediador do suporte social emocional nas relações do perdão disposicional com o florescimento no trabalho dos enfermeiros. As evidências reunidas demonstraram que o perdão disposicional e o suporte social emocional predisseram positivamente o florescimento no trabalho, conforme o esperado. Além disso, o suporte social emocional mediou parcialmente as relações do perdão disposicional com o florescimento no trabalho.

O modelo testado apresentou bons índices de ajuste e forneceu sustentação empírica adicional aos dois modelos que orientaram a pesquisa, quais sejam o Modelo de Demandas e Recursos do Trabalho (Bakker, \& Demerouti, 2007) e o Modelo de Worthington Jr et al. (2001). Tais resultados contribuíram para a extensão da rede nomológica do florescimento, especialmente no que diz respeito ao papel direto e mediador do suporte social em tal processo, nos profissionais de saúde e, mais especificamente, em enfermeiros, categoria de vital importância para o binômio saúde versus doença. No entanto, o modelo testado carece de investigações futuras, preferencialmente de natureza longitudinal, que se mostrem capazes de identificar relações de causalidade entre as variáveis investigadas.
No que diz respeito às aplicações práticas, a elaboração de programas de intervenção que desenvolvam tanto a prática de estender o perdão por parte da vítima quanto a prática de buscar o perdão por parte do ofensor, facilitando assim a reconciliação entre ambas as partes, pode se revelar útil. De igual modo, um plano de assistência ao enfermeiro que forneça aconselhamento ou ajuda tanto para as vítimas de uma ofensa interpessoal, quanto para os ofensores, também poderá ser proveitoso e estimular o florescimento do enfermeiro (Palanski, 2012).

Outrossim, o desenvolvimento de modelos de intervenções de natureza preventiva, que viabilizem mudanças na organização do trabalho do enfermeiro capazes de facilitar o florescimento dessa categoria profissional em seu contexto laboral, também poderão se revelar úteis. Nesse sentido, a adoção de políticas organizacionais voltadas à promoção do bom relacionamento entre os enfermeiros poderá impactar positivamente seu florescimento, visto que quando esses profissionais percebem que seu trabalho lhes dá suporte social, eles tendem a vivenciar maior bem-estar. A implementação de tais medidas reveste-se da capacidade potencial de contribuir não apenas para aumentar o florescimento no trabalho dos enfermeiros, mas também de seus colegas e superiores, o que indubitavelmente irá também se reverter em ganhos para suas organizações empregadoras e a sociedade em geral.

\section{Referências}

Ali, N. A. M., \& Panatik, S. A. (2013). The relationships between work values and work-related attitude: The role of social support as moderator. Journal of Social \& Development Sciences, 4(8), 369-375.

Al-Sabeelah, A. M. S., Alraggad, F. E. A., \& Ameerh, O. A. (2014). The relationship between forgiveness and personality traits, mental health among sample jordanian university students. International Journal of Education and Research, 2(9), 217-228.

Andrade, T., Hoch, R. E. E., Vieira, K. M., \& Rodrigues, C. M. C. (2012). Síndrome de burnout e suporte social no trabalho: A percepção dos profissionais de enfermagem de hospitais públicos e privados. Organizações \& Sociedade, 19(61), 231-251. https://doi.org/10.1590/S1984-92302012000200004

Bakker, A. B. (2011). An evidence-based model of work engagement. Current Directions in Psychological Science, 20(4), 265-269. https://doi.org/10.1177/0963721411414534

Bakker, A. B., \& Demerouti, E. (2007). The job demands-resources model: State of the art. Journal of Managerial Psychology, 22(3), 309-328. https://doi.org/10.1108/02683940710733115

Bakker, A. B., Demerouti, E., \& Schaufeli, W. B. (2003). Dual processes at work in a call centre: An application of the job demands-resources model. European Journal of Work and Organizational Psychology, 12(4), 393-417. https://doi.org/10.1080/13594320344000165

Berry, J. W., Worthington Jr., E. L., Parrott III, L., O’Connor, L. E., \& Wade, N. G. (2001). Dispositional forgivingness: Development and construct validity of the transgression narrative test of forgivingness (TNTF). Personality and Social Psychology Bulletin, 27(10), 1277-1290. https://doi.org/10.1177/01461672012710004. 
Berry, J. W., Worthington Jr., E. L., O’Connor, L. E., Parrott III, L., \& Wade, N. G. (2005). Forgivingness, vengeful rumination, and affective traits. Journal of Personality, 73(1), 183-226. https://doi.org/10.1111/j. 1467-6494.2004.00308.x

Burke, R. J., Moodie, S., Dolan, S., \& Fiksenbaum, L. (2012). Job demands, social support, work satisfaction and psychological well-being among nurses in Spain. ESADE Business School Research Paper, (233). Recuperado de https://papers.ssrn.com/sol3/papers.cfm?abstract_id=2117051

Byrne, B. M. (2001). Structural equation modeling with AMOS: Basic concepts, applications and programming. Mahwah, NJ: Lawrence Erlbaum.

Carver, C. S., \& Connor-Smith, J. (2010). Personality and coping. Annual Review of Psychology, 61, 679-704. https:// doi.org/10.1146/annurev.psych.093008.100352

Chaves, S. M. S. (2017). O impacto do perdão disposicional e do suporte social sobre o florescimento no trabalho de enfermeiros (Dissertação de Mestrado não publicada). Universidade Salgado de Oliveira, Niterói, RJ.

Cohen, S. (2004). Social relationships and health. American Psychologist, 59(8), 676-684. https://doi. org/10.1037/0003-066X.59.8.676

Crawford, E. R., Lepine, J. A., \& Rich, B. L. (2010). Linking job demands and resources to employee engagement and burnout: A theoretical extension and meta-analytic test. Journal of Applied Psychology, 95(5), 834-848. https:// doi.org/10.1037/a0019364

Damasceno, A. C., Campos, M. C. O., Falco, S., Souza, V. M., \& Penna, A. B. (2009). O enfermeiro frente a humanização e as relações interpessoais no ambiente laboral. Trabalho de Conclusão de Curso em Enfermagem, Faculdade de Ciências da Saúde de Barbacena, Universidade Presidente Antônio Carlos, Barbacena, MG. Recuperado de http://www.unipac.br/site/bb/tcc/tcc-dcc3220ee9875decabca1732e356cld8.

De Jonge, J., \& Schaufeli, W. B. (1998). Job characteristics and well-being: A test of Warr's vitamin model in health care workers using structural equation modelling. Journal of Organizational Behavior, 19(4), 387-407. https:// doi.org/10.1002/(SICI) 1099-1379(199807)19:4<387::AID-JOB851>3.0.CO;2-9

Deci, E. L., \& Ryan, R. M. (2008). Hedonia, eudaimonia, and well-being: An introduction. Journal of Happiness Studies, 9(1), 1-11. https://doi.org/10.1007/s10902-006-9018-1

Demerouti, E., Bakker, A. B., Nachreiner, F., \& Schaufeli, W. B. (2001). The job demands-resources model of burnout. Journal of Applied Psychology, 86(3), 499-512. https:// doi.org/10.1037/0021-9010.86.3.499

Dessen, M. C., \& Paz, M. G. T. (2010). Bem-estar pessoal nas organizações: O impacto de configurações de poder e características de personalidade. Psicologia: Teoria e Pesquisa, 26(3), 549-556. https://doi.org/10.1590/S010237722010000300018

Diener, E., \& Suh, E. M. (1997). Measuring quality of life: Economic, social and subjective indicators. Social Indicators Research, 40(1-2), 189-216. https://doi.org/10.1023/A:1006859511756

Diener, E., Wirtz, D., Tov, W., Kim-Prieto, C., Choi, D., Oishi, S.,\& Biswas-Diener, R. (2010). New well-being measures: Short scales to assess flourishing and positive and negative feelings. Social Indicators Research, 97, 143-156. https://doi.org/10.1007/s11205-009-9493-y10.1007/s11205-009-9493-y

Ducharme, L. J., \& Martin, J. K. (2000). Unrewarding work, coworker support and job satisfaction: A test of the buffering hypothesis. Work and Occupations, 27(2), 223-243. https://doi.org/10.1177/0730888400027002005

Dutton, J., Roberts, L., \& Bednar, J. (2010). Prosocial practices, positive identity, and flourishing at work. In: S. Donaldson, M. Csikszentmihalyi, \& J. Nakamura (Eds.), Applied positive psychology: Improving everyday life, schools, work, health, and society (pp. 155-170). New York, NY: Routledge.

Estivalete, V. F. B., Andrade, T., Faller, L. P., Stefanan, A. A., \& Souza, D. L. (2013). Suporte social e suporte organizacional como antecedentes de bem-estar no trabalho: A perspectiva dos colaboradores de uma empresa de logística ferroviária. Trabalho apresentado no XXXVII Encontro da ANPAD, Rio de Janeiro, RJ.

Ferreira, M. C., Silva, A. P. C., Fernandes, H. A., \& Almeida, S. P. (2008). Desenvolvimento e validação de uma escala de afetos no trabalho (ESAFE). Avaliação Psicológica, 7(2), 143-150.

Fornell, C., \& Larcker, D. F. (1981). Evaluating structural equations models with unobservable variables and measurement error. Journal of Marketing, 18(1), 39-50. https://doi.org/10.2307/3151312 
Gomes, A. R., Cruz, J. F., \& Cabanelas, S. (2009). Estresse ocupacional em profissionais de saúde: Um estudo com enfermeiros portugueses. Psicologia: Teoria e Pesquisa, 25(3), 307-318. https://doi.org/10.1590/S010237722009000300004

Gomide Junior, S., Guimarães, L. C., \& Damásio, L. F. Q. (2004). Construção e validação de um instrumento de medida de percepção de suporte social no trabalho. In: Resumos, Seminário de Pesquisa do Grupo Interinstitucional de Pesquisa em Bem-Estar, Suporte Social e Trabalho. Uberlândia, MG: Universidade Federal de Uberlândia.

Gouveia, V. V., Freires, L. A., Gouveia, R. S. V., Souza Filho, J. F., Souza, R. V. L., \& Monteiro, R. P. (2015). Escala de disposição para perdoar: Estrutura, consistência interna e invariância fatorial. Estudos de Psicologia, 32(2), 151-161. https://doi.org/10.1590/0103-166X2015000200001

Hobfoll, S. E. (2002). Social and psychological resources and adaptation. Review of General Psychology, 6(4), 307324. https://doi.org/10.1037/1089-2680.6.4.307

Hobfoll, S. E., Johnson, R. J., Ennis, N., \& Jackson, A. P. (2003). Resource loss, resource gain, and emotional outcomes among inner city women. Journal of Personality and Social Psychology, 84(3), 632-643. https://doi. org/10.1037/0022-3514.84.3.632

Hombrados-Mendieta, I., \& Cosano-Rivas, F. (2013). Burnout, workplace support, job satisfaction and life satisfaction among social workers in Spain: A structural equation model. International Social Work, 56(2), 228-246. https://doi.org/10.1177/0020872811421620

Humphrey, S. E., Nahrgang, J. D., \& Morgeson, F. P. (2007). Integrating motivational, social, and contextual work design features: A meta-analytic summary and theoretical extension of the work design literature. The Journal of Applied Psychology, 92(5), 1332-1356. https://doi.org/10.1037/0021-9010.92.5.1332

Huppert, F. A., \& So, T. T. C. (2009). What percentage of people in Europe are flourishing and what characterises them? Trabalho apresentado no OCDE/SQOLS Measuring subjective well-being: An opportunity for NSO's?, Florença, Italia. Recuperado de https://www.researchgate.net/publication/253825861_What_percentage_of_people_in_ Europe_are_flourishing_and_what_characterises_them

Keyes, C. L. M. (2002). The mental health continuum: From languishing to flourishing in life. Journal of Health and Social Research, 43(2), 207-222.

Keyes, C. L. M. (2006). Subjective well-being in mental health and human development research worldwide: An introduction. Social Indicators Research, 77(1), 1-10. https://doi.org/10.1007/s11205-005-5550-3

Keyes, C. L. M., Shmotkin, D., \& Ryff, C. D. (2002). Optimizing well-being: The empirical encounter of two traditions. Journal of Personality and Social Psychology, 82(6), 1007-1022. https://doi.org/10.1037/0022-3514.82.6.1007

Lawler-Row, K. A., \& Piferi, R. L. (2006). The forgiveness personality: Describing a life-well lived? Personality and Individual Differences, 41(6), 1009-1020. https:// doi.org/10.1016/j.paid.2006.04.007

Leite, N. M. B. (2007). Síndrome de burnout e relações sociais no trabalho: Um estudo com professores da educação básica. Dissertação de Mestrado, Instituto de Psicologia, Universidade de Brasília, Brasília, DF. Recuperado de http://repositorio.unb.br/handle/10482/3261.

Locke, E. A. (1976). The nature and causes of job satisfaction. In: M. D. Dunnette (Ed.), Handbook of industrial and organizational psychology (pp. 1297-1249). Chicago, IL: Rand McNally.

Mendonça, H., Caetano, A., Ferreira, M. C., Sousa, I. F., \& Silva, A. J. (2014). Florescimento e afetos no trabalho. In: M. M. M. Siqueira (Ed.), Novas medidas de comportamento organizacional: Ferramentas de diagnóstico e de gestão (pp. 172-177). Porto Alegre, RS: Artmed.

Palanski, M. E. (2012). Forgiveness and reconciliation in the workplace: A multi-level perspective and research agenda. Journal of Business Ethics, 109(3), 275-287. https://doi.org/10.1007/s10551-011-1125-1

Paschoal, T., Torres, C. V., \& Porto, J. B. (2010). Felicidade no trabalho: Relações com suporte organizacional e suporte social. Revista de Administração Contemporânea, 14(6), 1054-1072. https://doi.org/10.1590/S141565552010000700005

Reed, G. L., \& Enright, R. D. (2006). The effects of forgiveness therapy on depression, anxiety, and posttraumatic stress for women after spousal emotional abuse. Journal of Consulting and Clinical Psychology, 74(5), 920-929. https://doi.org/10.1037/0022-006X.74.5.920

Roberts, R. C. (1995). Forgivingness. American Philosophical Quarterly, 32(4), 289-306. 
Rodríguez-Carvajal, R., Moreno-Jiménez B., Rivas-Hermosilla, S., Álvarez-Bejarano, A., \& Sanz-Vergel, A. I. (2010). Positive psychology at work: Mutual gains for individuals and organizations. Revista de Psicología del Trabajo y de las Oganizaciones, 26(3), 235-253. https://doi.org/10.5093/tr2010v26n3a7

Rodriguez, M. S., \& Cohen, S. (1998). Social support. Encyclopedia of Mental Health, 3, 535-544.

Ryan, R. M., \& Deci, E. L. (2001). On happiness and human potentials: A review of research on hedonic and eudaimonic well-being. Annual Review of Psychology, 52, 141-166. https://doi.org/10.1146/annurev.psych.52.1.141

Ryff, C. D. (1989). Happiness is everything, or is it? Explorations on the meaning of psychological well-being. Journal of Personality and Social Psychology, 57, 1069-1081. https://doi.org/10.1037/0022-3514.57.6.1069

Ryff, C. D. (1995). Psychological well-being in adult life. Current Directions in Psychological Science, 4(4), 99-104. https://doi.org/10.1111/1467-8721.ep10772395

Ryff, C. D., \& Keyes, C. L. M. (1995). The structure of psychological well-being revisited. Journal of Personality and Social Psychology, 69(4), 719-727. https://doi.org/10.1037/0022-3514.69.4.719

Ryff, C. D., \& Singer, B. H. (2008). Know thyself and become what you are: A eudaimonic approach to psychological well-being. Journal of Happiness Studies, 9(1), 13-39. https://doi.org/10.1007/s10902-006-9019-0

Salanova, M., Bakker, A. B., \& Llorens, S. (2006). Flow at work: Evidence for an upward spiral of personal and organizational resources. Journal of Happiness Studies, 7(1), 1-22. https://doi.org/10.1007/s10902-005-8854-8

Sarason B., \& Duck S. (2001). Personal relationship: Implications for clinical and community psychology. Chichester: John Wiley \& Sons.

Schaufeli, W. B., \& Bakker, A. B. (2004). Job demands, job resources, and their relationship with burnout and engagement: A multi-sample study. Journal of Organizational Behavior, 25(3), 293-315. https://doi.org/10.1002/job.248

Tamayo, M. R., \& Tróccoli, B. T. (2002). Exaustão emocional: Relações com a percepção de suporte organizacional e com as estratégias de coping no trabalho. Estudos de Psicologia, 7(1), 37-42. https://doi.org/10.1590/S1413294X2002000100005

Temoshok, L. R., \& Chandra, P. S. (2000). The meaning of forgiveness in a specific situational and cultural context: Persons living with HIV/AIDS in India. In: M. E. McCullough, K. I. Pargament, \& C. E. Thoresen (Eds.), Forgiveness: Theory, research, and practice (pp. 41-64). New York, NY: Guilford.

Temoshok, L. R., \&Wald, R. L. (2005). Forgiveness and health in persons living with HIV/AIDS. In: E. L. Worthington Jr. (Ed.), Handbook of forgiveness (pp. 335-348). New York, NY: Routledge.

Thompson, L. Y., Snyder, C. R., Hoffman, L., Michael, S. T., Rasmussen, H. N., Bilings, L. S. et al. (2005). Dispositional forgiveness of self, others, and situations. Journal of Personality, 73(2), 313-360. https://doi.org/10.1111/j. 1467-6494.2005.00311.x

Toussaint, L., \& Friedman, P. (2008). Forgiveness, gratitude, and well-being: The mediating role of affect and beliefs. Journal of Happiness Studies, 10, 635-654. https://doi.org/10.1007/s10902-008-9111-8

Toussaint, L., \&Webb, J. R. (2005). Theoretical and empirical connections between forgiveness, mental health, and well-being. In: E. L. Worthington Jr. (Eds), Handbook of forgiveness (pp. 349-362). New York, NY: Routledge.

Van den Heuvel, M. (2013). Adaptation to organizational change: The role of meaningmaking and other psychological resources. Utrecht: Ipskamp Drukkers.

Van Yperen, N. W. (1998). Informational support and burnout: The moderating effect of self-efficacy. Journal of Ocupational and Organizational Psychology, 71(1), 29-33. https://doi.org/10.1111/j.2044-8325.1998.tb00660.x

Vazquez, A. C. S., Freitas, C. P. P., Cyrre, A., Hutz, C., \& Schaufeli, W. B. (2018). Evidências de validade da versão brasileira da escala de workaholism (DUWAS) e sua versão breve (DUWAS-10). Avaliação Psicológica, 17(1), 69-78. https://doi.org/10.15689/ap.2017.1701.08.13288

Vazquez, A. C. S., Magnan, E. S., Pacico, J. C., Hutz, C., \& Schaufeli, W. B. (2015). Adaptation and validation of the brazilian version of the Utrecht work engagement scale. Psico-USF, 20(2), 207-217. https:// doi.org/10.1590/141382712015200202

Webb, J. R., Hirsh, J. K., Visser, P. L. \& Brewer, K. G. (2013). Forgiveness and health: Assessing the mediating effect of health behavior, social support, and interpersonal functioning. The Journal of Psychology, 147(5), 391-414. https:// doi.org/10.1080/00223980.2012.700964 
Worthington Jr, E. L., Berry, J. W., \& Parrott III, L. (2001). Unforgiveness, forgiveness, religion, and health. In: T. G. Plante, \& A. C. Sherman (Eds.), Faith and health: Psychological perspectives (pp. 107-138). New York, NY: Guilford.

Worthington Jr., E. L., \& Drinkard, D. T. (2000). Promoting reconciliation through psychoeducational and therapeutic interventions. Journal of Marital and Family Therapy, 26(1), 93-101. https://doi.org/10.1111/j.1752-0606.2000. tb00279.x

Worthington Jr, E. L., \& Scherer, M. (2004). Forgiveness is an emotion-focused coping strategy that can reduce health risks and promote health resilience: Theory, review, and hypotheses. Psychology \& Health, 19(3), 385-405. https://doi.org/10.1080/0887044042000196674

Worthington Jr., E. L., \& Wade, N. G. (1999). The psychology of unforgiveness and forgiveness and implications for clinical practice. Journal of Social and Clinical Psychology, 18(4), 385-418. https://doi.org/10.1521/ jscp.1999.18.4.385

Worthington Jr., E. L., Witvliet, C. V. O., Pietrini, P., \& Miller, A. J. (2007). Forgiveness, health, and well-being: A review of evidence for emotional versus decisional forgiveness, dispositional forgiveness, and reduced unforgiveness. Journal of Behavioral Medicine, 30(4), 291-302. https://doi.org/10.1007/s10865-007-9105-8

Xanthopoulou, D., Bakker, A. B., Demerouti, E., \& Schaufeli, W. B. (2007). The role of personal resources in the job demands-resources model. International Journal of Stress Management, 14(2), 121-141. https://doi. org/10.1037/1072-5245.14.2.121

Zacher, H., Jimmieson, N. L., \& Bordia, P. (2014). Time pressure and coworker support mediate the curvilinear relationship between age and occupational well-being. Journal of Occupational Health Psychology, 19(4), 462-475. https://doi.org/10.1037/a0036995

Zhu, H. (2015). Social support and affect balance mediate the association between forgiveness and life satisfaction. Social Indicators Research, 124(12), 671-681. https:/ /doi.org/10.1007/s11205-014-0790-8

\section{Solange Maia da Silva Chaves}

Mestre em Psicologia pela Universidade Salgado de Oliveira (Universo). Brasil. Graduada em Psicologia pela Universo.

E-mail: solangemaia@uol.com.br

http://orcid.org/0000-0002-0305-9935

\section{Maria Cristina Ferreira}

Professora titular e coordenadora do Programa de Pós-Graduação em Psicologia da Universidade Salgado de Oliveira (Universo). Brasil.

E-mail: mcris@centroin.com.br

http://orcid.org/0000-0003-0752-6710

\section{Michelle Morelo Pereira}

Mestre em Psicologia e Doutoranda em Psicologia na Universidade Salgado de Oliveira (Universo). Brasil. Professora adjunta nas Faculdades Integradas Maria Thereza (Famath). Niterói - RJ. Brasil.

E-mail:mi_morelo@hotmail.com

http://orcid.org/0000-0003-2437-2071

\section{Clarissa Pinto Pizarro de Freitas}

Professora Titular do Programa de Pós-Graduação em Psicologia da Universidade Salgado de Oliveira (Universo). Brasil.

E-mail: freitas.cpp@gmail.com

http://orcid.org/0000-0002-2274-8728 
Recebido 31/08/2017

Reformulado 11/04/2018

Aceito 17/04/2018

Recived 08/31/2017

Reformated 04/11/2018

Approved 04/17/2018

Recibido 31/08/2017

Reformulado 11/04/2018

Aceptado 17/04/2018

Como citar: Chaves, S. M. S., Ferreira, M. C., Pereira, M. M., \& Freitas, C. P. P. (2019). Florescimento no Trabalho: Impacto do Perdão Disposicional e do Suporte Social Emocional. Psicologia: Ciência e Profissão, 39, 1-17. https://doi.org/10.1590/1982-3703003184816

How to cite: Chaves, S. M. S., Ferreira, M. C., Pereira, M. M., \& Freitas, C. P. P. (2019). Flourishing at Work: Impact of Dispositional Forgiveness and Emotional Social Support. Psicologia: Ciência e Profissão, 39, 1-17. https://doi.org/10.1590/1982-3703003184816

Cómo citar: Chaves, S. M. S., Ferreira, M. C., Pereira, M. M., \& Freitas, C. P. P. (2019). Florecimiento en el Trabajo: Impacto del Perdón Disposicional y del Soporte Social Emocional. Psicologia: Ciência e Profissão, 39, 1-17. https://doi.org/10.1590/1982-3703003184816 